\title{
MATRIX TRANSFORMATIONS OF POWER SERIES
}

\author{
DAVID BORWEIN AND AMNON JAKIMOVSKI
}

(Communicated by Andrew M. Bruckner)

\begin{abstract}
We consider the sequence of transforms $\left(g_{n}\right)$ of a power series $\sum_{n=0}^{\infty} a_{n} z^{n}$ given by $g_{n}(z):=\sum_{k=0}^{\infty} b_{n k} a_{k} z^{k}$. We establish necessary and sufficient conditions on the matrix $\left(b_{n k}\right)$ for the sequence $\left(g_{n}\right)$ to converge uniformly on compact subsets of the disk $D_{P}:=\{z:|z|<P\}$ to a function holomorphic on $D_{P}$.
\end{abstract}

\section{INTRODUCTION}

Suppose throughout that $0<P \leq \infty, 0<R<\infty$, and that all sequences and matrices are complex with indices running through $0,1,2, \ldots$ We make the following definitions:

$D_{P}$ is the disk $\{z:|z|<P\}$;

$\mathscr{E}$ is the set of all sequences a $\equiv\left(a_{n}\right)$ such that $\lim \left|a_{n}\right|^{\frac{1}{n+1}}=0$;

$\mathscr{E}^{\beta}$ is the set of all sequences $\mathrm{a} \equiv\left(a_{n}\right)$ such that $\lim \sup \left|a_{n}\right|^{\frac{1}{n+1}}<\infty$;

$\mathscr{E}_{R}$ is the set of all sequences $\mathrm{a} \equiv\left(a_{n}\right)$ such that $\sum_{n=0}^{\infty}\left|a_{n}\right| R^{n}<\infty$;

$\mathbf{A}_{R}$ is the set of all sequences $\mathbf{a} \equiv\left(a_{n}\right)$ such that $\lim \sup \left|a_{n}\right|^{\frac{1}{n+1}}=\frac{1}{R}$;

It will follow from the lemma (below) that $\mathscr{E}^{\beta}$ is the $\beta$-dual of $\mathscr{E}^{R}$.

The following are the first three of eight theorems we shall prove concerning matrix transformations of power series.

Theorem 1. A matrix $\mathbf{B} \equiv\left(b_{n k}\right)$ has the property that whenever the sequence $\mathbf{a} \equiv\left(a_{n}\right) \in \mathscr{E}_{R}$ the sequence of functions $\left(g_{n}\right)$ given by

$$
g_{n}(z):=\sum_{k=0}^{\infty} b_{n k} a_{k} z^{k}, \quad n=0,1, \ldots,
$$

converges uniformly on every compact subset of $D_{P}$, each power series $\sum_{k=0}^{\infty} b_{n k} a_{k} z^{k}$ being convergent on $D_{P}$, if and only if

(i) $\lim _{n \rightarrow \infty} b_{n k}=: b_{k}$ for $k=0,1, \ldots$;

(ii) $\sup _{n \geq 0, k \geq 0}\left|b_{n k}\right|\left(\frac{p}{R}\right)^{k}<\infty$ for each positive $p<P$.

And then $\lim _{n \rightarrow \infty} g_{n}(z)=\sum_{k=0}^{\infty} b_{k} a_{k} z^{k}$ on $D_{P}$.

Received by the editors October 7, 1992 and, in revised form, January 13, 1993.

1991 Mathematics Subject Classification. Primary 47B37, 47A30; Secondary 40G05.

Key words and phrases. Nörlund, matrix transforms, power series.

This research was supported in part by the Natural Sciences and Engineering Research Council of Canada. 
Theorem 2. A matrix $\mathbf{B} \equiv\left(b_{n k}\right)$ has the property that whenever the sequence $\mathbf{a} \equiv\left(a_{n}\right) \in \mathbf{A}_{R}$ the sequence of functions $\left(g_{n}\right)$ given by

$$
g_{n}(z):=\sum_{k=0}^{\infty} b_{n k} a_{k} z^{k}, \quad n=0,1, \ldots,
$$

converges uniformly on every compact subset of $D_{P}$, each power series $\sum_{k=0}^{\infty} b_{n k} a_{k} z^{k}$ being convergent on $D_{P}$, if and only if

(i) $\lim _{n \rightarrow \infty} b_{n k}=: b_{k}$ for $k=0,1, \ldots$;

(ii) $\sup _{n \geq 0, k \geq 0}\left|b_{n k}\right|\left(\frac{p}{R}\right)^{k}<\infty$ for each positive $p<P$.

And then $\lim _{n \rightarrow \infty} g_{n}(z)=\sum_{k=0}^{\infty} b_{k} a_{k} z^{k}$ on $D_{P}$.

Theorem 3. A matrix $\mathbf{B} \equiv\left(b_{n k}\right)$ has the property that whenever the sequence $\mathbf{a} \equiv\left(a_{n}\right) \in \mathscr{E}$ the sequence of functions $\left(g_{n}\right)$ given by

$$
g_{n}(z):=\sum_{k=0}^{\infty} b_{n k} a_{k} z^{k}, \quad n=0,1, \ldots,
$$

converges uniformly on every compact subset of $D_{\infty}$, each power series $\sum_{k=0}^{\infty} b_{n k} a_{k} z^{k}$ being convergent on $D_{\infty}$, if and only if

(i) $\lim _{n \rightarrow \infty} b_{n k}=: b_{k}$ for $k=0,1, \ldots$;

(ii) $\left.\sup _{n \geq 0, k \geq 0} ! b_{n k}\right|^{\frac{1}{k+1}}<\infty$.

And then $\lim _{n \rightarrow \infty} g_{n}(z)=\sum_{k=0}^{\infty} b_{k} a_{k} z^{k}$ on $D_{\infty}$.

These theorems show that if the series-to-sequence transform given by $\mathbf{B}$ is regular, then it is necessary in each case that $\lim _{n \rightarrow \infty} b_{n k}=b_{k}=1$ for $k=0,1, \ldots$, and this in turn implies that $P \leq R$ in Theorems 1 and 2 (i.e., the sequence $\left(g_{n}\right)$ cannot converge uniformly in any disk $D_{P}$ with $\left.P>R\right)$. Regular sequence-to-sequence transforms of power series have been considered by Peyerimhoff [5] and Luh [4] among others. One of the novel features of our approach is that we deal with series-to-sequence transforms rather than sequence-to-sequence transforms.

Let $\left(B_{n}\right)$ be a sequence of nonzero complex numbers. The associated Nörlund series-to-sequence matrix $\mathbf{N}_{B}$ is the triangular matrix $\left(b_{n k}\right)$ with

$$
b_{n k}:= \begin{cases}\frac{B_{n-k}}{B_{n}} & \text { if } 0 \leq k \leq n, \\ 0 & \text { otherwise. }\end{cases}
$$

The following theorem is an immediate consequence of Theorem 2. The case $R=1$ of Theorem KS is due to Karin Stadtmüller [6, Theorem 5]. Her method of proof is different from and more complicated than the one developed below.

Theorem KS. The Nörlund matrix $\mathbf{N}_{B}$ has the property that whenever the sequence $\mathbf{a} \equiv\left(a_{n}\right) \in \mathbf{A}_{R}$ the sequence of functions $\left(g_{n}\right)$ given by

$$
g_{n}(z):=\frac{1}{B_{n}} \sum_{k=0}^{n} B_{n-k} a_{k} z^{k}, \quad n=0,1, \ldots,
$$

converges uniformly on every compact subset of $D_{P}$, if and only if

$$
\lim _{n \rightarrow \infty} \frac{B_{n-1}}{B_{n}}=b \text { with }|b|=\frac{R}{P} .
$$

And then $\lim _{n \rightarrow \infty} g_{n}(z)=\sum_{k=0}^{\infty} a_{k}(b z)^{k}$ on $D_{P}$. 
Note. In view of Theorem 1 , Theorem $\mathrm{KS}$ remains true if $\mathbf{A}_{R}$ is replaced by $\mathscr{E}_{R}$.

\section{A PRELIMINARY RESULT}

Lemma. A sequence b has the property that $\sum_{n=0}^{\infty} b_{n} a_{n}$ is convergent for each $\mathbf{a} \in \mathscr{E}$ if and only if $\mathbf{b} \in \mathscr{E}^{\beta}$.

Proof. Sufficiency. If $\mathbf{b} \in \mathscr{E}^{\beta}$, then there exists a positive number $M$ such that $\left|b_{n}\right| \leq M^{n+1}$ for $n=0,1, \ldots$ Hence, if $a \in \mathscr{E}$, then $\sum_{k=0}^{\infty}\left|b_{k} a_{k}\right| \leq$ $M \sum_{k=0}^{\infty}\left|a_{k}\right| M^{k}<\infty$.

Necessity. Assume b $\notin \mathscr{E}^{\beta}$, i.e., $\lim \sup \left|b_{n}\right|^{\frac{1}{n+1}}=\infty$. Then there exists a strictly increasing sequence of positive integers $\left(n_{j}\right)$ such that $0<\left|b_{n_{j}}\right|^{\frac{1}{n_{j}+1}} \rightarrow$ $\infty$. Choose

$$
a_{n}:= \begin{cases}\frac{1}{\sqrt{\left|b_{n}\right|}} & \text { if } n=n_{j} \\ 0 & \text { otherwise. }\end{cases}
$$

Then

$$
\left|a_{n}\right|^{\frac{1}{n+1}}= \begin{cases}\left(\frac{1}{\left|b_{n}\right|^{\frac{1}{n+1}}}\right)^{1 / 2} & \text { if } n=n_{j} \\ 0 & \text { otherwise. }\end{cases}
$$

Hence $\lim \left|a_{n}\right|^{\frac{1}{n+1}}=0$, so a $\in \mathscr{E}$. But

$$
\left|b_{n_{j}} a_{n_{j}}\right|=\sqrt{\left|b_{n_{j}}\right|}=\left(\left|b_{n_{j}}\right|^{\frac{1}{n_{j}+1}}\right)^{\frac{n_{j}+1}{2}} \rightarrow \infty \text { as } j \rightarrow \infty,
$$

and therefore $\sum_{n=0}^{\infty} b_{n} a_{n}$ is not convergent.

\section{Proofs of Theorems 1,2 , AND 3}

Proof of Theorem 1. Sufficiency. We assume that

$$
\begin{cases}\lim _{n \rightarrow \infty} b_{n k}=: b_{k} & \text { for } k=0,1, \ldots ; \\ M(p):=\sup _{n \geq 0, k \geq 0}\left|b_{n k}\right|\left(\frac{p}{R}\right)^{k}<\infty & \text { for } 0<p<P .\end{cases}
$$

Let $a \in \mathscr{C}_{R}$. We have, for $n=0,1, \ldots$ and $|z| \leq p<P$,

$$
\left|\sum_{k=0}^{\infty} b_{n k} a_{k} z^{k}\right| \leq \sum_{k=0}^{\infty}\left|b_{n k}\right|\left|a_{k}\right| p^{k} \leq M(p) \sum_{k=0}^{\infty}\left|a_{k}\right| R^{k}<\infty .
$$

Hence the functions $g_{n}(z):=\sum_{k=0}^{\infty} b_{n k} a_{k} z^{k}$ are holomorphic and uniformly bounded on $D_{p}$. Also $g_{n}^{(k)}(0)=k ! b_{n k} a_{k} \rightarrow k ! b_{k} a_{k}$ as $n \rightarrow \infty$ for $k=$ $0,1, \ldots$. Further, from Cauchy's inequalities for the coefficients of power series we get that, for $|z| \leq p_{1}<p<P, n=0,1, \ldots$, and $k=0,1, \ldots$,

$$
\left|b_{n k} a_{k} z^{k}\right| \leq M(p, \mathbf{a})\left(p_{1} / p\right)^{k}, \quad \text { where } M(p, \mathbf{a}):=\sup _{n \geq 0} \max _{|z|=p}\left|g_{n}(z)\right|<\infty \text {. }
$$

Therefore, by the Weierstrass M-test, $\lim _{n \rightarrow \infty} g_{n}(z)=\sum_{k=0}^{\infty} b_{k} a_{k} z^{k}$ on $D_{P}$, and the sequence $\left(g_{n}\right)$ is uniformly convergent on compact subsets of $D_{P}$. 
Necessity. Let $a_{k}:=1 /\left((k+1)^{2} R^{k}\right)$ for $k=0,1,2, \ldots$ Since $\mathbf{a} \in \mathscr{E}_{R}$, our assumption is that the series $g_{n}(z):=\sum_{k=0}^{\infty} b_{n k} a_{k} z^{k}$ converges on $D_{P}$ and that the sequence $\left(g_{n}\right)$ is uniformly convergent on $D_{p}$ for $0<p<P$. By the Weierstrass double-series theorem, $\lim _{n \rightarrow \infty} b_{n k} a_{k}$ exists for $k=0,1, \ldots$. Since $a_{k} \neq 0$ for $k=0,1, \ldots$, it follows that the condition

$$
\lim _{n \rightarrow \infty} b_{n k}=: b_{k} \text { for } k=0,1, \ldots
$$

must necessarily hold. Suppose now that $p$ and $\tilde{p}$ are fixed and $0<p<\tilde{p}<P$. Since the sequence $\left(g_{n}\right)$ is uniformly convergent on $\bar{D}_{\tilde{p}}$, the closure of $D_{\tilde{p}}$, we have, for $|z| \leq \tilde{p}$ and $n=0,1, \ldots$, that $\left|g_{n}(z)\right| \leq M(\tilde{p}, \mathbf{a})<\infty$. From Cauchy's inequalities for the coefficients of power series we get that

$$
\left|b_{n k} a_{k} \tilde{p}^{k}\right| \leq M(\tilde{p}, \mathbf{a}) \text { for } n=0,1, \ldots \text { and } k=0,1, \ldots,
$$

and hence that

$$
\sup _{n \geq 0, k \geq 0}\left|b_{n k}\right|\left(\frac{p}{R}\right)^{k} \leq M(\tilde{p}, \mathbf{a}) \sup _{k \geq 0}\left(\frac{p}{\tilde{p}}\right)^{k}(k+1)^{2}<\infty .
$$

Therefore the condition

$$
\sup _{n \geq 0, k \geq 0}\left|b_{n k}\right|\left(\frac{p}{R}\right)^{k}<\infty \text { for all positive } p<P
$$

is also necessary.

Proof of Theorem 2. Sufficiency. We assume that

$$
\begin{cases}\lim _{n \rightarrow \infty} b_{n k}=: b_{k} & \text { for } k=0,1, \ldots ; \\ M(p):=\sup _{n \geq 0, k \geq 0}\left|b_{n k}\right|\left(\frac{p}{R}\right)^{k}<\infty & \text { for } 0<p<P .\end{cases}
$$

Let $\mathbf{a} \in \mathbf{A}_{R}$. For $0<p<P$ choose $r$ so that $0<r<R$ and $\frac{p}{r}<\frac{P}{R}$. Now choose $p_{1}$ such that $0<p_{1}<P$ and $\frac{p}{r}=\frac{p_{1}}{R}$. We have, for $|z| \leq p$, that

$$
\begin{aligned}
\left|\sum_{k=0}^{\infty} b_{n k} a_{k} z^{k}\right| & \leq \sum_{k=0}^{\infty}\left|b_{n k}\right|\left|a_{k}\right| p^{k}=\sum_{k=0}^{\infty}\left|b_{n k}\right|\left(\frac{p}{r}\right)^{k}\left|a_{k}\right| r^{k} \\
& =\sum_{k=0}^{\infty}\left|b_{n k}\right|\left(\frac{p_{1}}{R}\right)^{k}\left|a_{k}\right| r^{k} \leq M\left(p_{1}\right) \sum_{k=0}^{\infty}\left|a_{k}\right| r^{k}<\infty .
\end{aligned}
$$

Hence the functions $g_{n}(z):=\sum_{k=0}^{\infty} b_{n k} a_{k} z^{k}$ are uniformly bounded on $D_{p}$ for $0<p<P$. Also $g_{n}^{(k)}(0)=k ! b_{n k} a_{k} \rightarrow k ! b_{k} a_{k}$ as $n \rightarrow \infty$ for $k=0,1, \ldots$. Further, from Cauchy's inequalities for the coefficients of power series we get that, for $|z| \leq p_{1}<p<P, n=0,1, \ldots$ and $k=0,1, \ldots$,

$$
\left|b_{n k} a_{k} z^{k}\right| \leq M(p, \mathbf{a})\left(p_{1} / p\right)^{k}, \quad \text { where } M(p, \mathbf{a}):=\sup _{n \geq 0} \max _{|z|=p}\left|g_{n}(z)\right|<\infty .
$$

Therefore, by the Weierstrass M-test, $\lim _{n \rightarrow \infty} g_{n}(z)=\sum_{k=0}^{\infty} b_{k} a_{k} z^{k}$ on $D_{P}$, and the sequence $\left(g_{n}\right)$ is uniformly convergent on compact subsets of $D_{P}$.

Necessity. Let $a_{k}:=1 / R^{k}$ for $k=0,1,2, \ldots$. Since $\mathbf{a} \in \mathbf{A}_{R}$, our assumption is that the series $g_{n}(z):=\sum_{k=0}^{\infty} b_{n k} a_{k} z^{k}$ converges on $D_{P}$ and that the 
sequence $\left(g_{n}\right)$ is uniformly convergent on $D_{p}$ for $0<p<P$. By the Weierstrass double-series theorem, $\lim _{n \rightarrow \infty} b_{n k} a_{k}$ exists for $k=0,1, \ldots$. Since $a_{k} \neq 0$ for $k=0,1, \ldots$, it follows that the condition

$$
\lim _{n \rightarrow \infty} b_{n k}=: b_{k} \text { for } k=0,1, \ldots
$$

must necessarily hold. Suppose now that $p$ is fixed and $0<p<P$. Since the sequence $\left(g_{n}\right)$ is uniformly convergent on $\bar{D}_{p}$, we have, for $|z| \leq p$ and $n=0,1, \ldots$, that $\left|g_{n}(z)\right| \leq M(p$, a) $<\infty$. From Cauchy's inequalities for the coefficients of power series we get that

$$
\left|b_{n k}\right|\left(\frac{p}{R}\right)^{k}=\left|b_{n k} a_{k} p^{k}\right| \leq M(p, \mathbf{a}) \text { for } n=0,1, \ldots \text { and } k=0,1, \ldots
$$

Therefore, the condition

$$
\sup _{n \geq 0, k \geq 0}\left|b_{n k}\right|\left(\frac{p}{R}\right)^{k}<\infty \text { for all positive } p<P
$$

is also necessary.

Proof of Theorem 3. Sufficiency. We assume that

$$
\left\{\begin{array}{l}
\lim _{n \rightarrow \infty} b_{n k}=b_{k} \text { for } k=0,1, \ldots, \\
M:=\sup _{n \geq 0, k \geq 0}\left|b_{n k}\right|^{\frac{1}{k+1}}<\infty
\end{array}\right.
$$

Let $\mathbf{a} \in \mathscr{E}$. We have, for $|z| \leq R<\infty$, that

$$
\left|\sum_{k=0}^{\infty} b_{n k} a_{k} z^{k}\right| \leq \sum_{k=0}^{\infty}\left|b_{n k}\right|\left|a_{k}\right| M^{k} \leq M \sum_{k=0}^{\infty}\left|a_{k}\right|(M R)^{k}<\infty .
$$

Hence the functions $g_{n}(z):=\sum_{k=0}^{\infty} b_{n k} a_{k} z^{k}$ are entire and are uniformly bounded on each closed disk $\bar{D}_{R}$. Also $g_{n}^{(k)}(0)=k ! b_{n k} a_{k} \rightarrow k ! b_{k} a_{k}$ as $n \rightarrow \infty$ for $k=0,1, \ldots$. Further, from Cauchy's inequalities for the coefficients of power series we get that, for $|z| \leq p<R, n=0,1, \ldots$ and $k=0,1, \ldots$,

$$
\left|b_{n k} a_{k} z^{k}\right| \leq M(R, \mathbf{a})\left(\frac{p}{R}\right)^{k}, \quad \text { where } M(R, \mathbf{a}):=\sup _{n \geq 0} \max _{|z|=R}\left|g_{n}(z)\right|<\infty .
$$

Therefore, by the Weierstrass M-test, $\lim _{n \rightarrow \infty} g_{n}(z)=\sum_{k=0}^{\infty} b_{k} a_{k} z^{k}$ on $D_{\infty}$, and the sequence $\left(g_{n}\right)$ is uniformly convergent on compact subsets of $D_{\infty}$.

Necessity. We assume that for each a $\in \mathscr{E}$ the series $g_{n}(z):=$ $\sum_{k=0}^{\infty} b_{n k} a_{k} z^{k}$ is convergent on $D_{\infty}$ and that the sequence $\left(g_{n}\right)$ is uniformly convergent on compact subsets of $D_{\infty}$. By the Weierstrass double-series theorem, $\lim _{n \rightarrow \infty} b_{n k} a_{k}$ exists for $k=0,1, \ldots$. Since there is an a $\in \mathscr{E}$ such that $a_{k} \neq 0$ for $k=0,1, \ldots$, it follows that the condition

$$
\lim _{n \rightarrow \infty} b_{n k}=: b_{k} \text { for } k=0,1, \ldots
$$

must necessarily hold.

Suppose that $\mathbf{a} \in \mathscr{E}$. Since the sequence $\left(g_{n}\right)$ is uniformly convergent on $D_{R}$, we have, for $|z| \leq R$ and $n=0,1, \ldots$, that $\left|g_{n}(z)\right| \leq M(R, \mathbf{a})<\infty$. From Cauchy's inequalities for the coefficients of power series we get that

$$
\left|b_{n k} a_{k} R^{k}\right| \leq M(R, \mathbf{a}) \text { for } n=0,1, \ldots \text { and } k=0,1, \ldots \text {. }
$$


Also, since $\sum_{k=0}^{\infty} b_{n k} a_{k}$ is convergent whenever $\mathbf{a} \in \mathscr{E}$, we have, by the lemma, that

$$
M_{n}:=\sup _{k \geq 0}\left|b_{n k}\right|^{\frac{1}{k+1}}<\infty \text { for } n=0,1, \ldots
$$

Assume now that

$$
\sup _{n \geq 0} \sup _{k \geq 0}\left|b_{n k}\right|^{\frac{1}{k+1}}=\sup _{n \geq 0} M_{n}=\infty
$$

This implies that there exists a strictly increasing sequence of positive integers $\left(n_{j}\right)$ such that $M_{n_{j}} \rightarrow \infty$. This in turn implies that there exists a sequence of nonnegative integers $\left(k_{j}\right)$ such that

$$
\text { (*) } \quad\left|b_{n_{j}, k_{j}}\right|^{\frac{1}{k_{j}+1}}>\frac{1}{2} M_{n_{j}} \rightarrow \infty \text { as } j \rightarrow \infty \text {. }
$$

We show now that the sequence $\left(k_{j}\right)$ is not bounded. Assume that it is bounded. Then there is a positive integer $k^{*}$ such that $0 \leq k_{j} \leq k^{*}$. Since $\lim _{n \rightarrow \infty} b_{n k}$ $=b_{k}$ for $k=0,1, \ldots, k^{*}$, it follows that the set of numbers $\left(b_{n k}\right)_{n \geq 0,0 \leq k \leq k^{*}}$ is bounded and hence that the set of numbers $\left(\left|b_{n k}\right|^{\frac{1}{k+1}}\right)_{n \geq 0,0 \leq k \leq k^{*}}$ is bounded. But this contradicts $(*)$. Therefore, the sequence $\left(k_{j}\right)$ is not bounded. We can suppose (by considering a subsequence if necessary) that the sequence is strictly increasing. Choose

$$
a_{k}:= \begin{cases}1 /\left(\left|b_{n_{j}, k}\right|\right)^{\frac{k+1}{2}} & \text { if } k=k_{j} \\ 0 & \text { otherwise }\end{cases}
$$

We then have

$$
\left|a_{k_{j}}\right|^{\frac{1}{k_{j}+1}}=\frac{1}{\sqrt{\left|b_{n_{j}, k_{j}}\right|}}<\left(\frac{1}{\frac{1}{2} M_{n_{j}}}\right)^{\frac{k_{j}+1}{2}} \rightarrow 0 \text { as } j \rightarrow \infty .
$$

Therefore $a \in \mathscr{E}$, but

$$
\left|b_{n_{j}, k_{j}}\right| a_{k_{j}}=\sqrt{\mid b_{n_{j}, k_{j}}} \rightarrow \infty \text { as } j \rightarrow \infty,
$$

which contradicts (1). Thus the condition

$$
\sup _{n \geq 0, k \geq 0}\left|b_{n k}\right|^{\frac{1}{k+1}}<\infty
$$

is also necessary.

\section{AdDitional theOREMS}

In this section we prove some theorems showing that the disk of convergence $D_{P}$ specified in Theorem 2 cannot be enlarged when the matrix $\mathbf{B}$ satisfies conditions (i) and (ii) of that theorem together with certain other conditions.

Theorem 4. Suppose that $P$ and $R$ are positive numbers, and that $\mathbf{B} \equiv\left(b_{n k}\right)$ is a normal infinite matrix (i.e., $b_{n k}=0$ for $k>n$ and $b_{n n} \neq 0$ ) satisfying

$$
M(p):=\sup _{n \geq 0, k \geq 0}\left|b_{n k}\right|\left(\frac{p}{R}\right)^{k}<\infty \text { for } 0<p<P .
$$


Then, for each $\mathbf{a} \in \mathbf{A}_{R}$ and each $R_{1} \geq P$,

$$
\limsup _{n \rightarrow \infty} \max _{|z|=R_{1}}\left|\sum_{k=0}^{n} b_{n k} a_{k} z^{k}\right|^{\frac{1}{n}} \leq \frac{R_{1}}{P}
$$

Proof. Choose $R_{1} \geq P$, and suppose a $\in \mathbf{A}_{R}$. Let $0<\lambda<1$, and take $p:=\lambda P$. Then $0<p<P$. Since $\lim \sup \left|a_{k}\right|^{\frac{1}{k+1}}=\frac{1}{R}$, there is a positive constant $c(\lambda)$ such that

$$
\left|a_{k}\right| \leq \frac{c(\lambda)}{(\lambda R)^{k}} \text { for } k \geq 0 .
$$

Now for $|z|=R_{1}$ we have

$$
\begin{aligned}
\left|\sum_{k=0}^{n} b_{n k} a_{k} z^{k}\right| & \leq \sum_{k=0}^{n}\left|b_{n k}\right|\left(\frac{p}{R}\right)^{k}\left|a_{k}\right| R^{k}\left(\frac{R_{1}}{p}\right)^{k} \\
& \leq M(p) c(\lambda) \sum_{k=0}^{n}\left(\frac{R}{\lambda R}\right)^{k}\left(\frac{R_{1}}{\lambda P}\right)^{k}=M(p) c(\lambda) \sum_{k=0}^{n}\left(\frac{R_{1}}{\lambda^{2} P}\right)^{k} .
\end{aligned}
$$

Since $R_{1} /\left(\lambda^{2} P\right)>R_{1} / P \geq 1$, it follows that

$$
\underset{n \rightarrow \infty}{\limsup } \max _{|z|=R_{1}}\left|\sum_{k=0}^{n} b_{n k} a_{k} z^{k}\right|^{\frac{1}{n}} \leq \lim _{n \rightarrow \infty}\left(\sum_{k=0}^{n}\left(\frac{R_{1}}{\lambda^{2} P}\right)^{k}\right)^{\frac{1}{n}}=\frac{R_{1}}{\lambda^{2} P} .
$$

Letting $\lambda \nearrow 1$ we get

$$
\underset{n \rightarrow \infty}{\limsup } \max _{|z|=R_{1}}\left|\sum_{k=0}^{n} b_{n k} a_{k} z^{n}\right|^{\frac{1}{n}} \leq \frac{R_{1}}{P} .
$$

Remark. Assume that a normal matrix B satisfies

$$
M(p):=\sup _{n \geq 0, k \geq 0}\left|b_{n k}\right|\left(\frac{p}{R}\right)^{k}<\infty \text { for } 0<p<P .
$$

Then

$$
\left|b_{n n}\right|^{\frac{1}{n}} \frac{p}{R} \leq M(p)^{\frac{1}{n}} \rightarrow 1 \text { as } n \rightarrow \infty,
$$

and hence

$$
\limsup _{n \rightarrow \infty}\left|b_{n n}\right|^{\frac{1}{n}} \leq \frac{R}{p} \quad \text { for each positive } p<P .
$$

Letting $p \nearrow P$ we get

$$
\limsup _{n \rightarrow \infty}\left|b_{n n}\right|^{\frac{1}{n}} \leq \frac{R}{P}
$$

This suggests that it is not inappropriate to impose the condition

$$
\lim _{n \rightarrow \infty}\left|b_{n n}\right|^{\frac{1}{n}}=\frac{R}{P},
$$

as we do in the following theorem. 
Theorem 5. Let B be a normal matrix. Suppose that

$$
\lim _{n \rightarrow \infty}\left|b_{n n}\right|^{\frac{1}{n}}=\frac{R}{P},
$$

where $P$ and $R$ are positive numbers. Then for each $\mathbf{a} \in \mathbf{A}_{R}$ and each $R_{1} \geq P$ we have

$$
\limsup _{n \rightarrow \infty} \max _{|z|=R_{1}}\left|\sum_{k=0}^{n} b_{n k} a_{k} z^{k}\right|^{\frac{1}{n}} \geq \frac{R_{1}}{P} .
$$

Proof. Assume that the conclusion of the theorem is not true. Then there is an $\mathrm{a}^{*} \in \mathbf{A}_{R}$ and an $R_{1} \geq P$ such that

$$
\limsup _{n \rightarrow \infty} \max _{|z|=R_{1}}\left|\sum_{k=0}^{n} b_{n k} a_{k}^{*} z^{k}\right|^{\frac{1}{n}}<\frac{R_{1}}{P} .
$$

Therefore, there exists a positive $\tilde{R}<R_{1}$ such that, for all $n$ sufficiently large,

$$
\max _{|z|=R_{1}}\left|\sum_{k=0}^{n} b_{n k} a_{k}^{*} z^{k}\right|^{\frac{1}{n}} \leq \frac{\tilde{R}}{P}, \text { and hence } \max _{|z|=R_{1}}\left|\sum_{k=0}^{n} b_{n k} a_{k}^{*} z^{k}\right| \leq\left(\frac{\tilde{R}}{P}\right)^{n} .
$$

Applying the Cauchy inequalities to the function $g_{n}(z):=\sum_{k=0}^{n} b_{n k} a_{k}^{*} z^{k}$ we get in particular that, for all large $n$,

$$
\left|b_{n n}\right|\left|a_{n}^{*}\right| R_{1}^{n} \leq\left(\frac{\tilde{R}}{P}\right)^{n} \text {, and therefore }\left|b_{n n}\right|^{\frac{1}{n}}\left|a_{n}^{*}\right|^{\frac{1}{n}} R_{1} \leq \frac{\tilde{R}}{P} .
$$

From the last inequality we get that

$$
\frac{\tilde{R}}{P} \geq \limsup _{n \rightarrow \infty}\left(\left|b_{n n}\right|^{\frac{1}{n}}\left|a_{n}^{*}\right|^{\frac{1}{n}} R_{1}\right)=R_{1} \lim _{n \rightarrow \infty}\left|b_{n n}\right|^{\frac{1}{n}} \cdot \limsup _{n \rightarrow \infty}\left|a_{n}^{*}\right|^{\frac{1}{n}}=\frac{R_{1}}{P} .
$$

But this is a contradiction since $0<\tilde{R}<R_{1}$. Hence the conclusion of the theorem must hold.

The next two theorems generalize results about regular and nonregular Nörlund matrices due respectively to Luh [3] and K. Stadtmüller [6, Theorems 6 and 7]. The first of these theorems, which follows immediately from Theorems 4 and 5, shows, inter alia, that the sequence $\left(g_{n}\right)$ specified in Theorem 2 cannot converge uniformly in any disk $D_{P_{1}}$ with $P_{1}>P$ when $\mathrm{B}$ is a normal matrix satisfying condition (ii) of Theorem 2 together with the diagonal condition of Theorem 5.

Theorem 6. Suppose that $P$ and $R$ are positive numbers and that $\mathbf{B}$ is a normal matrix satisfying

$$
M(p):=\sup _{n \geq 0, k \geq 0}\left|b_{n k}\right|\left(\frac{p}{R}\right)^{k}<\infty \text { for } 0<p<P \text { and } \lim _{n \rightarrow \infty}\left|b_{n n}\right|^{\frac{1}{n}}=\frac{R}{P} .
$$

Then, for each $\mathbf{a} \in \mathbf{A}_{R}$ and each $R_{1} \geq P$,

$$
\limsup _{n \rightarrow \infty} \max _{|z|=R_{1}}\left|\sum_{k=0}^{n} b_{n k} a_{k} z^{k}\right|^{\frac{1}{n}}=\frac{R_{1}}{P} \text {. }
$$

The next theorem shows that the circle $|z|=R_{1}$ in the conclusion of Theorem 6 can be replaced by any arc of that circle when condition (i) of Theorem 2 is also satisfied. 
Theorem 7. Suppose that $P$ and $R$ are positive numbers and that $\mathbf{B}$ is a normal matrix such that

$$
\begin{gathered}
\lim _{n \rightarrow \infty} b_{n k}=: b_{k} \quad \text { for } k=0,1, \ldots, \text { where } b_{k} \neq 0 \text { for } k>k^{*} ; \\
M(p):=\sup _{n \geq 0, k \geq 0}\left|b_{n k}\right|\left(\frac{p}{R}\right)^{k}<\infty \text { for } 0<p<P, \text { and } \lim _{n \rightarrow \infty}\left|b_{n n}\right|^{\frac{1}{n}}=\frac{R}{P} .
\end{gathered}
$$

Then, for each $\mathbf{a} \in \mathbf{A}_{R}$ and each $R_{1} \geq P$,

$$
\limsup _{n \rightarrow \infty} \max _{z \in \Gamma}\left|\sum_{k=0}^{n} b_{n k} a_{k} z^{k}\right|^{\frac{1}{n}}=\frac{R_{1}}{P},
$$

where $\Gamma$ is any closed non-trivial arc of $|z|=R_{1}$.

Proof. By Theorem 6 we know that

$$
\limsup _{n \rightarrow \infty} \max _{z \in \Gamma}\left|\sum_{k=0}^{n} b_{n k} a_{k} z^{k}\right|^{\frac{1}{n}} \leq \frac{R_{1}}{P} .
$$

Hence it is enough to prove that, for every $\mathbf{a} \in \mathbf{A}_{R}$,

$$
\limsup _{n \rightarrow \infty} \max _{z \in \Gamma}\left|\sum_{k=0}^{n} b_{n k} a_{k} z^{k}\right|^{\frac{1}{n}} \geq \frac{R_{1}}{P},
$$

which we now proceed to do.

Case 1. $R_{1}=P$ : Suppose (2) is not true. Then for some $\mathbf{a}^{*} \in \mathbf{A}_{R}$ we have

$$
\underset{n \rightarrow \infty}{\lim \sup } \max _{z \in \Gamma}\left|\sum_{k=0}^{n} b_{n k} a_{k}^{*} z^{k}\right|^{\frac{1}{n}}<\frac{R_{1}}{P}=1 .
$$

It follows that there exists a positive number $q<1$ such that, for all $n$ sufficiently large,

$$
\sup _{z \in \Gamma}\left|\sum_{k=0}^{n} b_{n k} a_{k}^{*} z^{k}\right|<q^{n} .
$$

Given $\epsilon>0$ we get from Theorem 6 that, for all $n$ sufficiently large,

$$
\max _{|z|=P}\left|\sum_{k=0}^{n} b_{n k} a_{k}^{*} z^{k}\right| \leq 2^{\epsilon n}
$$

For $0<r<P$ we have, by Nevanlinna's $N$-constants theorem (see [1, Theorem 18.3.3]), that there exists a positive number $\theta<1$ (depending on $r$ but not on $\epsilon$ ) such that, for all large $n$,

$$
\max _{|z|=r}\left|\sum_{k=0}^{n} b_{n k} a_{k}^{*} z^{k}\right| \leq\left(q^{\theta} 2^{(1-\theta) \epsilon}\right)^{n} .
$$

Since we can choose $\epsilon>0$ so small that $q^{\theta} 2^{(1-\theta) \epsilon}<1$, it follows that

$$
\max _{|z|=r}\left|\sum_{k=0}^{n} b_{n k} a_{k}^{*} z^{k}\right| \rightarrow 0 \quad \text { as } n \rightarrow \infty .
$$


By the Weierstrass double-series theorem we get that

$$
0=\lim _{n \rightarrow \infty} b_{n k} a_{k}^{*}=b_{k} a_{k}^{*} \quad \text { for } k=0,1, \ldots
$$

Since $\mathbf{a}^{*} \in \mathbf{A}_{R}$, we have that $a_{k}^{*} \neq 0$ for some $k>k^{*}$. Hence $b_{k}=0$ for such a $k$. But this contradicts the assumption that $b_{k} \neq 0$ for $k>k^{*}$. Therefore (2) must hold when $R_{1}=P$.

Case 2. $R_{1}>P$ : Assume that (2) is not true. Then there exists a sequence $\mathbf{a}^{*} \in \mathbf{A}_{R}$ and a number $\tilde{R}$ such that $P<\tilde{R}<R_{1}$ and

$$
\underset{n \rightarrow \infty}{\limsup } \max _{z \in \Gamma}\left|\sum_{k=0}^{n} b_{n k} a_{k}^{*} z^{k}\right|^{\frac{1}{n}} \leq \frac{\tilde{R}}{P}
$$

Hence given $\epsilon>0$ we have, for all sufficiently large $n$,

$$
\max _{z \in \Gamma}\left|z^{-n} \sum_{k=0}^{n} b_{n k} a_{k}^{*} z^{k}\right| \leq\left(\frac{\tilde{R}}{P} \cdot \frac{1}{R_{1}}\right)^{n} 2^{\epsilon n}=\left(\frac{\tilde{R}}{R_{1}}\right)^{n}\left(\frac{2^{\epsilon}}{P}\right)^{n} .
$$

Further, from Theorem 6 we get that, for all large $n$,

$$
\max _{|z|=P}\left|z^{-n} \sum_{k=0}^{n} b_{n k} a_{k}^{*} z^{k}\right| \leq\left(\frac{2^{\epsilon}}{P}\right)^{n}
$$

and

$$
\max _{|z|=R_{1}}\left|z^{-n} \sum_{k=0}^{n} b_{n k} a_{k}^{*} z^{k}\right| \leq\left(\frac{2^{\epsilon}}{P}\right)^{n} \text {. }
$$

Let $g_{n}(z):=\sum_{k=0}^{n} b_{n k} a_{k}^{*} z^{k}$, and let $P<r<R_{1}$. Then, by Nevanlinna's $N$ constants theorem, there exist positive constants $\theta_{1}, \theta_{2}, \theta_{3}$ (depending on $r$ but not on $\epsilon$ ) such that $\theta_{1}+\theta_{2}+\theta_{3}=1$ and

$$
\max _{|z|=r}\left|\frac{g_{n}(z)}{z^{n}}\right| \leq\left(\frac{\tilde{R}}{R_{1}} \frac{2^{\epsilon}}{P}\right)^{n \theta_{1}}\left(\frac{2^{\epsilon}}{P}\right)^{n \theta_{2}}\left(\frac{2^{\epsilon}}{P}\right)^{n \theta_{3}}=\left(\frac{\tilde{R}}{R_{1}}\right)^{n \theta_{1}}\left(\frac{2^{\epsilon}}{P}\right)^{n}
$$

for all sufficiently large $n$. Hence, choosing $\epsilon>0$ so small that $\left(\tilde{R} / R_{1}\right)^{\theta_{1}} 2^{\epsilon}$ $<1$, we get

$$
\underset{n \rightarrow \infty}{\lim \sup } \max _{|z|=r}\left|g_{n}(z)\right|^{\frac{1}{n}} \leq\left(\frac{\tilde{R}}{R_{1}}\right)^{\theta_{1}} 2^{\epsilon} \frac{r}{P}<\frac{r}{P} .
$$

Since $r>P$, the last inequality contradicts the conclusion of Theorem 5 . Hence (2) must hold when $R_{1}>P$.

The next theorem deals with the possibility of pointwise convergence of the sequence $\left(g_{n}(z)\right)$ specified in Theorem 2 outside the convergence disk $D_{P}$. It generalizes results due to Lejá [2] and Stadtmüller [6, Theorem 8] about regular and nonregular Nörlund matrices respectively. Both authors mistakenly assumed that their proofs were valid when, in the notation of the following theorem, $R=1$ and sequence $\left(a_{n}\right)$ is bounded. The example $a_{n}:=1 /(n+1)$ shows that their method of proof cannot be used in this case. The difficulty is avoided in our Theorem 8 by the imposition of the limsup condition. 
Theorem 8. Suppose that $P$ and $R$ are positive numbers and that $\mathbf{B}$ is a normal matrix such that

$$
\begin{gathered}
\lim _{n \rightarrow \infty} b_{n k}=: b_{k} \text { for } k=0,1, \ldots, \text { where } b_{k} \neq 0 \text { for } k>k^{*} ; \\
M(p):=\sup _{n \geq 0, k \geq 0}\left|b_{n k}\right|\left(\frac{p}{R}\right)^{k}<\infty \text { for } 0<p<P ; \quad \lim _{n \rightarrow \infty}\left|b_{n n}\right|^{\frac{1}{n}}=\frac{R}{P},
\end{gathered}
$$

and

$$
\left|b_{n k}\right| \leq c(\tilde{R})\left|b_{n n}\right|\left(\frac{P}{\tilde{R}}\right)^{n-k} \quad \text { for } 0<\tilde{R}<R \text { and } 0 \leq k \leq n .
$$

Suppose that $\mathbf{a} \in \mathbf{A}_{R}$ and that $\lim \sup _{n \rightarrow \infty}\left|a_{n}\right| R^{n}>0$. Let

$$
g_{n}(z):=\sum_{k=0}^{n} b_{n k} a_{k} z^{k}
$$

Then lim $\sup _{n \rightarrow \infty}\left|g_{n}(z)\right|^{\frac{1}{n}} \leq 1$ for at most a finite number of points $z$ satisfying $|z|>P_{1}>P$, and hence, in particular, the sequence $\left(g_{n}\right)$ can converge at most at a finite number of points $z$ satisfying $|z|>P_{1}>P$.

Proof. Let $c_{n}:=a_{n} R^{n}$ where $\mathbf{a} \in \mathbf{A}_{R}$, and let $\lim \sup _{n \rightarrow \infty}\left|c_{n}\right|>c>0$. Define

$$
M:= \begin{cases}1 & \text { if } \sup _{n \geq 0}\left|c_{n}\right|=\infty, \\ c^{-1} \sup _{n \geq 0}\left|c_{n}\right| & \text { otherwise. }\end{cases}
$$

By considering the unbounded monotonic sequence $\left(d_{n}\right)$ where $d_{n}:=$ $\max _{0 \leq k \leq n}\left|c_{k}\right|$ when $\max _{n \geq 0}\left|c_{n}\right|=\infty$, we see that there is a strictly increasing sequence of positive integers $\left(n_{k}\right)$ integers such that

$$
\left|c_{n}\right| \leq M\left|c_{n_{k}}\right| \text { for } 0 \leq n<n_{k} \text {, and }\left|c_{n_{k}}\right|>c .
$$

Since $\lim \sup _{n \rightarrow \infty}\left|c_{n}\right|^{\frac{1}{n}}=1$, we have

$$
1 \geq \limsup _{k \rightarrow \infty}\left|c_{n_{k}}\right|^{\frac{1}{n_{k}}} \geq \liminf _{k \rightarrow \infty}\left|c_{n_{k}}\right|^{\frac{1}{n_{k}}} \geq \lim _{k \rightarrow \infty} c^{\frac{1}{n_{k}}}=1,
$$

so $\lim _{k \rightarrow \infty}\left|c_{n_{k}}\right|^{\frac{1}{n_{k}}}=1$. Whenever $c_{n} \neq 0$, let

$$
\tilde{g}_{n}(z):=\sum_{j=0}^{n} \frac{b_{n j}}{b_{n n}} \frac{c_{j}}{c_{n}}\left(\frac{z}{R}\right)^{j-n}=\frac{g_{n}(z)}{b_{n n} c_{n}(z / R)^{n}} ;
$$

and let

$$
h_{k}(w):=\tilde{g}_{n_{k}}\left(\frac{1}{w}\right)
$$

Assume that $z^{*}$ is a point such that $\left|z^{*}\right|>P_{1}$ and $\lim \sup _{n \rightarrow \infty}\left|g_{n}\left(z^{*}\right)\right|^{\frac{1}{n}} \leq 1$. Since

$$
\begin{aligned}
\lim _{k \rightarrow \infty}\left|b_{n_{k}, n_{k}} c_{n_{k}}\left(\frac{z^{*}}{R}\right)^{n}\right|^{\frac{1}{n}} & =\lim _{k \rightarrow \infty}\left|b_{n_{k}, n_{k}}\right|^{\frac{1}{n_{k}}} \cdot \lim _{k \rightarrow \infty}\left|c_{n_{k}}\right|^{\frac{1}{n_{k}}} \cdot \frac{\left|z^{*}\right|}{R} \\
& \geq \frac{R}{P} \frac{P_{1}}{R}=\frac{P_{1}}{P}>1,
\end{aligned}
$$


it follows from (3) and (4) that $\lim \sup _{k \rightarrow \infty}\left|\tilde{g}_{n_{k}}\left(z^{*}\right)\right|^{\frac{1}{n_{k}}}<1$ and hence that

$$
\lim _{k \rightarrow \infty} h_{k}\left(w^{*}\right)=0 \quad \text { where } w^{*}:=1 / z^{*} .
$$

Suppose $|w| \leq 1 / P^{*}$ where $P_{1}>P^{*}>P$. Then we have, for $0<\tilde{R}<R$,

$$
\left|h_{k}(w)\right| \leq \sum_{j=0}^{n_{k}} c(\tilde{R})\left(\frac{P}{\tilde{R}}\right)^{n_{k}-j} M\left(\frac{R}{P^{*}}\right)^{n_{k}-j}=c(\tilde{R}) M \sum_{j=0}^{n_{k}}\left(\frac{P}{P^{*}} \frac{R}{\tilde{R}}\right)^{n_{k}-j} .
$$

Choose $\tilde{R}<R$ so close to $R$ that $0<\frac{P}{P^{*}} \frac{R}{\tilde{R}}<1$. Then

$$
\left|h_{k}(w)\right| \leq \frac{c(\tilde{R}) M}{1-\frac{P}{P^{*}} \frac{R}{\tilde{R}}}<\infty \text { for }|w| \leq \frac{1}{P^{*}}<\frac{1}{P} \text { and } k \geq 0 .
$$

This means that the sequence $\left(h_{k}(w)\right)$ is uniformly bounded for $|w| \leq 1 / P^{*}$. Suppose now that there are infinitely many points $z_{r}$ with $\left|z_{r}\right|>P_{1}>P^{*}$ such that $\lim \sup _{n \rightarrow \infty}\left|g_{n}\left(z_{r}\right)\right|^{\frac{1}{n}} \leq 1$. Then by (5)

$$
\lim _{k \rightarrow \infty} h_{k}\left(w_{r}\right)=0 \text { for } w_{r}:=1 / z_{r}
$$

By Vitali's theorem (see [7, Theorem 5.2.1]) the sequence $\left(h_{k}(w)\right)$ converges uniformly to 0 on compact subsets of $D_{\frac{1}{P^{*}}}$. In particular,

$$
0=\lim _{k \rightarrow \infty} h_{n_{k}}(0)=1,
$$

which is a contradiction. Hence there are at most finitely many points $z$ such that $|z|>P_{1}$ and $\lim \sup _{n \rightarrow \infty}\left|g_{n}(z)\right|^{\frac{1}{n}} \leq 1$.

\section{Construction}

In this section we construct a Nörlund matrix $\mathbf{N}_{B}$ satisfying the hypotheses of Theorem 8 with $P=1$ such that the corresponding sequence of transforms $\left(g_{n}\right)$ of the power series $\sum_{k=0}^{\infty}(z / R)^{k}$ converges at $N$ points outside the convergence disk $D_{1}$.

Let $p(z)$ be a polynomial of degree $N$ defined by

$$
p(z):=\sum_{k=0}^{\infty} p_{k} z^{k}:=\left(z+\alpha_{1}\right)\left(z+\alpha_{2}\right) \cdots\left(z+\alpha_{N}\right),
$$

where $0<\alpha_{1}<\alpha_{2}<\cdots<\alpha_{N}<1$. Define the Nörlund matrix $\mathbf{N}_{B} \equiv\left(b_{n k}\right)$ by setting

$$
b_{n k}:=\frac{B_{n-k}}{B_{n}} \text { for } 0 \leq k \leq n, \text { where } B_{n}:=\frac{1}{R^{n}} \sum_{k=0}^{n} p_{k} .
$$


Then, for $a_{k}:=1 / R^{k}, w=1 / z$, and $n \geq N$,

$$
\begin{aligned}
g_{n}(z): & =\sum_{k=0}^{n} b_{n k} a_{k} z^{k}=\frac{1}{B_{n}} \sum_{k=0}^{n} B_{n-k}\left(\frac{z}{R}\right)^{k} \\
& =\frac{z^{n}}{B_{n} R^{n}} \sum_{k=0}^{n} B_{k}(R w)^{k}=\frac{z^{n}}{B_{n} R^{n}} \sum_{k=0}^{n} w^{k} \sum_{j=0}^{k} p_{j} \\
& =\frac{z^{n}}{B_{n} R^{n}} \sum_{j=0}^{n} p_{j} \sum_{k=j}^{n} w^{k}=\frac{z^{n}}{B_{n} R^{n}} \sum_{j=0}^{n} p_{j} \frac{w^{j}-w^{n+1}}{1-w} \\
& =\frac{z^{n}}{B_{n} R^{n}} \frac{p(w)}{1-w}-\frac{w}{1-w} .
\end{aligned}
$$

Hence, for every $n \geq N$, we have $g_{n}(z)=z /(1-z)$ whenever $p(w)=0$, and this occurs when $z=-1 / a_{k}, k=1,2, \ldots, N$.

\section{ACKNOWLEDGMENT}

We are grateful to the referee for suggesting the versions of the proofs in $\S 3$ of the necessity parts of Theorems 1 and 2. Our original proofs were longer and more complicated.

\section{REFERENCES}

1. E. Hille, Analytic function theory, Blaisdell, New York, 1963.

2. M. F. Lejá, Sur la sommation des séries entières par la méthode des moyennes, Bull. Sci. Math. (2) 54 (1930), 239-245.

3. W. Luh, Über die Nörlund-Summierbarkeit von Potenzreihen, Period. Math. Hungar. 5 (1974), 47-60.

4. _ Summierbarkeit von Potenzreihen-notwendige Bedingungen, Mitt. Math. Sem. Giessen 113 (1974), 48-67.

5. A. Peyerimhoff, Lectures on summability, Lecture Notes in Math., vol. 107, Springer-Verlag, New York, 1969.

6. K. Stadtmüller, Summability of power series by non-regular Nörlund methods, J. Approx. Theory 68 (1991), 33-44.

7. E. C. Titchmarsh, The theory of functions, Oxford Univ. Press, London, 1947.

Department of Mathematics, University of Western Ontario, London, Ontario, CANADA N6A 5B7

E-mail address: dborrein@uro.ca

School of Mathematical Sciences, Tel Aviv University, Tel Aviv, Israel

E-mail address: jakimovemath.tau.ac.il 